\title{
Los estudiantes de CICOM de la Universidad del Altiplano. Una cartografía de su identidad ${ }^{*}$
}

\author{
Michel Montiel Meléndez ${ }^{1}$
}

\begin{abstract}
Resumen
La presente investigación buscó conocer quiénes son los universitarios de la licenciatura en Ciencias de la Comunicación de la Universidad del Altiplano, sobre todo, cómo conforman su identidad universitaria, se trata de un estudio con fundamentos epistémicos soportados en la fenomenología-hermenéutica, en particular, su diseño corresponde a un estudio de caso con enfoque etnográfico, mediante el cual, se buscó el encuentro con los actores, a partir de su contexto y, de las experiencias en el mismo. Los hallazgos permitieron establecer una tipología en cuanto a los estudiantes que conforman la comunidad CICOM-UDA, además de conocer las razones por las cuales optaron estudiar esta licenciatura y los motivos que los llevó a matricularse en una universidad privada, de igual forma, los imaginarios sociales sobre dos condiciones; la primera, por pertenecer a este programa educativo; y la segunda, por ser parte de una institución privada, además, de identificar cuáles son las expectativas de éxito profesional de los alumnos.

Abstract

This thesis had the objective of studying how bachelor students of Communication Sciences in 'Universidad del Altiplano' construct their university identity. This study has an epistemological foundation in phenomenology and hermeneutics. The method is a case study with an ethnographic design, which sought to observe the actors in their own context and the experiences on it. The results allowed to propose a typology of the students from the community CICOM-UDA, as well as to know the reasons why they chose this program and the motivations to enroll in this private university. We were also able to detect the social imaginaries on two conditions: first, by being part of the program; and the second, in their role as part of the private institution. And finally, we were able to establish what are their expectations regarding professional success.
\end{abstract}

Palabras Clave

Identidad, Universitarios, Imaginarios, CICOM

Keywords

Identity, University Students, Imaginary, CICOM

*Tercer lugar del Concurso de Trabajos Recepcionales en Comunicación CONEICC 2018. Universidad del Altiplano. Asesor: Dr. Adrián Hernández Vélez

${ }^{1}$ Autor para correspondencia: michel.montiel09@gmail.com

\section{Introducción}

El interés por conocer el tema de la identidad de los estudiantes de la Licenciatura en Ciencias de la Comunicación (CICOM) apareció entre los escasos diálogos cargados de reflexión epistémica y metodológica, los cuales motivaron este trabajo, en el que, la identidad no sólo constituyó el objeto de estudio, sino también una ventana de acceso a la reflexión filosófica soportada en la relación entre el sujeto y su yo, es decir, el sujeto y su rol en la institución en la que se forma.

En México, destacan las investigaciones desarrolladas por De Garay (2012) quien analizó el caso de los universitarios mexicanos con relación a su perfil socioeconómico, intereses de pertenencia y construcción de identidad institucional. También, se cuenta con las aportaciones de Lozano y 
Rodríguez (2005) quienes asumen reflexiones colocadas en la misma línea para el caso de la UPN. Por su parte, Molina (2012) y Sartorello (2013) plantean su reflexión con los estudiantes de las universidades interculturales y para el caso de una institución particular, Basaldúa (2013) abordó la situación de la Universidad Iberoamericana-Puebla.

En conjunto, en todos los trabajos mencionados se reflexiona sobre la identidad de los universitarios, sus motivos académicos, procedencia y factores que definen su estancia en la universidad, sin embargo, son pocos los trabajos en esta línea; la Universidad del Altiplano (UDA) no cuenta con investigaciones de esta naturaleza, por esta razón, conocer y comprender quiénes conforman su comunidad estudiantil resulta un reto que fue asumido como objeto de estudio, en este marco, desde la perspectiva de los estudiantes, se buscó comprender su identidad, responder cuáles son los imaginarios que existen por cursar estudios en una institución privada, qué significa ser universitario, cuáles son las expectativas de éxito profesional, así como determinar los ejes que orientan la construcción de su identidad universitaria y profesionista.

La trascendencia de la investigación radica en responder a la ausencia de estudios de esta naturaleza en las instituciones privadas de Tlaxcala, por lo que su desarrollo permitió comprender a la comunidad de CICOM-UDA, aportar al estado del conocimiento y coadyuvar al análisis institucional sobre la vida interna de los universitarios.

\section{Fundamentos Teóricos}

Ha sido tanto lo escrito respecto al tema de identidad que de pronto no se logra comprender por qué en contextos concretos suele reducirse a su ínfima delimitación conceptual, como si los factores que se entretejen para dotarlo de sentido en la vida de facto quedaran anulados entre palabras. Esta es la razón por la cual se optó por construir un debate inicial sobre su conformación, no sólo como tema, sino en general, como un campo del conocimiento que trasciende esferas etnográficas, históricas e incluso sociales, entre otras.

Para ello, fueron trazados tres horizontes disciplinares, a través de los cuales, es orientada la reflexión; desde una perspectiva sociológica se señala a la identidad como la construcción de una narrativa mediada por la interacción del individuo con la sociedad. En cuanto a la postura psicológica, esta la aborda a partir de las características similares compartidas entre un individuo y los otros, mientras que, para la filosófica, es argumentada como un proceso mediante el cual el sujeto se asume de manera consciente dentro de un contexto particular.

Giménez (1997) refiere a la identidad como algo distinguible, es decir, esa característica particular de cada individuo que logra una diferenciación de los otros, a diferencia de Giddens (2002) que plantea la identidad con una correlación en la modernidad, Giménez afirma que la identidad es un elemento importante de la cultura, viéndola como una parte subjetiva en función de la distinguibilidad, así el autor atribuye esta característica a la identidad, y su aportación se coloca en otro sentido cuando la define como parte de la cultura en la que se desenvuelven los agentes de manera individual o colectiva.

Mientras que desde una perspectiva psicológica la identidad basa sus intereses en cambios culturales y modificaciones en la sociedad, así como en las transformaciones en los individuos, además, en este enfoque, es posible centrar la atención en dos tipos de identidades; "identidades: individual y colectiva (CIP-FUHEM, 2005)." Esto implica, que conocer la identidad individual es un trabajo laborioso y complejo ya que con el paso del tiempo, se han inaugurado nuevas alternativas de comportamiento y reglas de conducta, lo cual, atendiendo a sus reflexiones y a la dinámica de cambio social, permite precisar a la identidad como un conjunto de rasgos de un individuo o un grupo, por lo regular, vinculados a un sistema de símbolos y valores, los cuales, bajo un proceso de decodificación, son comprendidos, lo que les permite que cumplan su función en situaciones cotidianas, generando reacciones, además de que orientan la forma de pensar, actuar e interactuar; es decir, se trata de un proceso en constante recreación.

Por último, desde una perspectiva filosófica, en esta, Larrain (2003) parte de la premisa de no considerar a la identidad como una esencia con la que se nace, por el contrario, la aborda como un proceso de definición del ser en sí mismo con relación a los otros, es decir, como la capacidad de considerarse parte de una narrativa, que emerge, dadas las relaciones sociales que detonan interacciones y que a su vez son parte de la cultura a la que pertenecen. 
Asimismo señala el autor, la cultura es otro aspecto importante que se debe tratar para comprender lo que en sí representa la identidad, por cultura se debe entender a las formas simbólicas, mientras que la identidad es la manera en que estas formas se movilizan en la interacción para construir una narrativa.

En términos generales, la identidad desde una perspectiva sociológica es considerada como una característica adaptable que otorga un valor de representación al ser humano, el cual, permite la diferenciación entre los sujetos de la misma especie, dotando así de sentido su pertenencia a un grupo determinado, mientras que, abordado desde la psicología, la identidad resulta ser la definición de sí mismo con relación a los demás, a partir de un conjunto de significados de un sistema de símbolos y valores, en un sentido filosófico, la identidad radica en tejer una línea delgada, pero perceptible, entre el otro y uno mismo, el sentido de la alteridad está en reconocerse en el otro, pero también en establecer bordes finos entre él y uno, lo cual implica que uno es en tanto el otro es.

\section{Arquitectura metodológica}

Fue desarrollado un estudio cualitativo cuyo soporte epistémico radicó en una visión fenomenológica-hermenéutica. El diseño corresponde a un estudio de caso con enfoque etnográfico, soportado en cuatro núcleos temáticos: identidad universitaria, identidad CICOM, imaginarios sociales sobre la condición de estudiante de instituciones privadas y expectativas de éxito profesional.

El encuentro con los informantes se sostuvo a partir de grupos focales; para ello, fueron convocados estudiantes que cursaban el primero, tercero, quinto y séptimo cuatrimestre. Previamente, fue definida una matriz de temáticas-preguntas a través de la cual se siguió el curso de cuatro reuniones. Las opiniones vertidas fueron registradas en un formato doble; grabación de voz autorizada por los informantes y capturadas en texto.

Destacan dos procesos en el tratamiento de los datos; conforme al primero, el encuentro con los actores ocurrió en sus aulas y el diálogo desarrollado, permitió la comprensión e interpretación de sus palabras bajo los principios hermenéuticos, no obstante, como recurso complementario, posteriormente se abordó un proceso mediado por un sentido técnico, conforme al cual, fue empleado el software MaxQDA.

En el software se registró el sistema de núcleos problemáticos y fueron cargadas las entrevistas. El análisis desarrollado, permitió obtener matrices de fragmentos con los hallazgos generados en cada categoría hermenéutica, así fue posible identificar puntos de consistencia e inconsistencia que perfilaron la identidad de los estudiantes.

\section{Resultados}

Los resultados están organizados a partir de los cuatro ejes de análisis definidos en el diseño del estudio: identidad universitaria, identidad CICOM, imaginarios sociales sobre la condición de estudiante de instituciones privadas y expectativas de éxito profesional. A continuación, son presentados los hallazgos que permitieron las entrevistas realizadas, para lo cual, se recuperan fragmentos de lo expresado por los estudiantes.

\subsection{Identidad universitaria}

En este núcleo los testimonios permitieron establecer, los ejes bajo los cuales los estudiantes conforman su identidad universitaria, el primero radica en el significado que le atribuyen a la condición de universitario, en cuanto a este, fue posible construir una tipología. De acuerdo con el segundo eje, este se centra en los factores que contribuyeron para la elección de la universidad, en este sentido, el motivo de elección como un factor de satisfacción para su estancia en la institución. El tercero, recupera elementos que permiten sentirse parte de la UDA, la vida universitaria como un pilar fundamental para la construcción de su identidad. El cuarto eje, refiere a la importancia de los docentes como un referente de motivación para los estudiantes, conforme a los cuales, fue posible establecer una tipología.

... ser universitario, implica tener un cierto amor, por decirlo así, a tu escuela, [... ] en una forma muy coloquial decimos "ponerte la camiseta", es reflejar que tú eres tu universidad, o sea, donde te pares, siempre vas a tener renombre tanto bueno como malo, yo siento que ser universitario también es eso, sentirte parte de acá, porque literalmente es tu casa, tu segundo hogar, pasas la 
mayoría del tiempo acá, entonces deja que hagas tareas y todo eso, hasta como te vistas, siéntete parte de ella, siente que lo que estás haciendo es para un bien para ti, pero, también para reflejar, a futuro vas a ser la imagen de lo que fue tu hogar durante el periodo de escuela [... ] (S5, E CICOM 5)

En este sentido, tres de las posturas coinciden en que ser universitario va más allá de cursar estudios en una institución de educación superior y aunque son múltiples los factores señalados que intervienen para la auto denominación de esta condición, las experiencias son fundamentales en este espacio.

En lo que corresponde a la elección de universidad es una decisión determinante en la vida de los universitarios, también es un factor importante que coadyuva a su satisfacción para poderse identificar con la institución a la que se encuentran inscritos y en este sentido, son pocos quienes colocan como su primera opción a la UDA, no obstante, para quienes no fue su primera opción, resulta un factor de motivación estudiar la carrera que anhelaban para sentirse parte de la institución.

Para comprender la identidad universitaria, es necesario ahondar en tema de cultura, en este sentido, como referente inmediato y como definición de cultura retomada por lo que plantea Larrain (2003) la vida universitaria es un pilar importante en la construcción de los estudiantes, se trata de un espacio en el que los alumnos realizan diversos intercambios de símbolos mediante los cuales, se comunican entre sí $\mathrm{y}$, sobre todo, comparten cualquier tipo de experiencias, sin embargo, existen factores que no ayudan de manera proactiva al crecimiento de su vida universitaria, tal como destacaron los universitarios, entre estos se encuentran; la necesidad de pensar en una forma distinta a la organización académico-administrativa, también en la urgente necesidad de promover una verdadera extensión universitaria y, finalmente, la no menos urgente necesidad de construir la identidad universitaria no sólo en una visión endogámica atrapada en el programa de adscripción, sino, en una identidad institucional.

Conforme al docente como un referente de motivación, este ocupa un lugar importante como actor educativo, no sólo en las instituciones a las que se encuentra adscrito, sino también en el peso que tiene en la vida de los estudiantes y sobre todo, como afirma Rama (2011) el papel que desarrolla como factor de influencia para el aprendizaje de los alumnos, en este sentido, también es colocado como un referente para el desarrollo profesional de los futuros egresados y tal como señala Díaz Barriga y Hernández (2002) el docente también es un ser motivador que tiene como objetivo, entre otros, generar un interés en el alumnado por alguna actividad académica que coadyuve a su desarrollo personal y social.

... yo no tengo a ningún profesor que diga ok, este profesor es muy bueno, quiero que me ayude, que me asesore o así, la verdad en mi caso yo no tengo a ninguno, pero creo que tal vez en un futuro, con los profesores que vaya conociendo, encuentre a alguien que me guste lo que hace $y$ que me apasione lo que hace, que haga lo mismo que él hace, puede ser, por el momento yo no encuentro a ninguno. (S12, E CICOM 3)

En cuanto a lo observado, fue posible clasificar a los docentes que conforman la institución en dos tipos; profesores de casa y profesores invitados. Los primeros refieren a los profesores que cuentan con tiempo completo en la universidad, quizá no exclusivos de la carrera en CICOM, pero si con una carga curricular constante, cuatrimestre con cuatrimestre, cuentan con antigüedad en la institución, sus bases, no siempre son en educación, además de que la mayoría de los docentes frente a grupo en el área son profesores de casa, y estos se desarrollan profesionalmente en algún medio de comunicación, esto en consistencia con la tradición que remite a su razón de ser, el máximo grado académico que se presenta es maestría.

Por otro lado, a los profesores invitados, se trata de los docentes que forman parte de la matrícula de profesores, sin embargo, no cuentan con un tiempo completo y su carga curricular puede variar, dependiendo de sus propios intereses, horarios, así como de las asignaturas disponibles, son docentes que cuentan con un campo profesional destacado, en ocasiones su interés de participación es mínimo, pero con firmeza, pues como tal, se desempeñan en otras instituciones tales como BUAP, UATx, UDLAP, UPAEP, y UVT por ello, les resulta complicado responder a ciertas políticas internas; el máximo grado académico que presentan es de doctorado.

A manera de cierre, la identidad universitaria se construye con diversos factores, entre estos, es posible referir a tres; 
la cultura, el sentido de pertenencia y los otros, para los estudiantes de la UDA, resulta complicado la construcción y consolidación de su identidad al no ser esta su primera opción, y sobre todo, al no encontrar en la institución una vida cultural y deportiva que coadyuve a este proceso, sin embargo, son alumnos comprometidos con sus estudios quienes buscan generar este propio vínculo con la universidad y de este modo dotar de sentido su estadía universitaria.

\subsection{Identidad CICOM}

Para este núcleo se establecen dos de los ejes sobre los cuales, los alumnos soportan la construcción de su identidad CICOM, el primero radica en las razones por las cuales optaron por estudiar la Licenciatura en Ciencias de la Comunicación; sus motivos y razones; en cuanto al segundo, este se centra en los imaginarios sociales que existen sobre estudiar este programa educativo, y en particular, lo que será su profesión.

Si bien la elección de la universidad es un factor importante en la formación de un universitario, la elección de una carrera profesional es otro, incluso primordial, se trata de lo que se estudiará durante un periodo determinado y, más allá, lo que contribuirá a su desarrollo profesional, las razones pueden ser diversas, sin embargo, los motivos de elección radican también en la satisfacción que se tiene con la misma.

Entre los motivos sobre los cuales fundamentaron esta importante decisión es posible referir a cuatro; el primero, radica en el campo profesional, alumnos que decidieron estudiar CICOM esperando a futuro desempeñarse en medios de comunicación; el segundo, se centra en estudiar una carrera afín conforme a lo que sería su primera opción, pues no cuentan con el capital suficiente para sostener una licenciatura especializada en otras áreas; el tercero, refiere en considerar a la profesión una "todología", estudiantes que optaron por CICOM bajo la idea de ser una licenciatura con un perfil de egreso amplio, y por último; el cuarto motivo se coloca en estudiar CICOM como tradición familiar, se trata de alumnos que soportaron su decisión en los referentes que tienen desde su hogar.

Elegí la carrera porque me gusta el periodismo, bueno, mi papá es periodista, la verdad desde muy chico me di cuenta lo que él hacía y por eso me llamó la atención esta carrera, pero igual yo también pensé que sólo era periodismo, pero no, pero el campo es enorme, no nada más es escribir, también, hay muchas cosas como administración, cosas que a lo mejor no le entiendes ahorita, pero después vas a ver y sí, sí te ayuda mucho. (S3, E CICOM 3)

La preparación profesional en comunicación ha sido centro de diferentes percepciones, se trata de los imaginarios que existen en torno a esta $\mathrm{y}$, sobre todo, en los futuros profesionistas, en este sentido, existen múltiples definiciones para el concepto imaginario, sin embargo, se recurre a la precisión del concepto de Castoriadis (2002) quien, por imaginario social, refiere a una representación mental que es producto del reflejo de la realidad basado en un conjunto de significaciones propias.

En tónica similar, los estudiantes señalan tres ideas constantes sobre las cuales, es construido el imaginario del comunicólogo; en primera instancia, es recurrente la asociación de la carrera con el periodismo, pero sobre todo, con el homicidio hacia estos profesionistas, fenómeno que se ha presentado en los últimos años, en este sentido, los alumnos afirman que al menos personas de su contexto inmediato, vinculan su futura profesión con la muerte, lo cual, resulta un tanto lógico, pues de acuerdo al semanario Proceso (2016), México ocupa el tercer lugar de periodistas asesinados, sin embargo, este imaginario radica en la relación de estudiar ciencias de la comunicación con la formación de periodista.

Conforme a la segunda idea, esta radica en considerar a la profesión como una de las carreras más mal pagadas, en este sentido, la mayoría de las posturas asumidas por su entorno familiar no es aprobatoria, pues se parte de la idea de que se encuentran estudiando una licenciatura que no les asegurará un futuro laboral provechoso y mucho menos el éxito, al no ser un programa educativo como lo es derecho o medicina, por mencionar algunos, incluso, hay quienes afirman que sus padres preferirían que siguieran con la tradición familiar, es decir, optar por la misma profesión o al menos una similar.

Mi mamá siempre me decía que estudiara enfermería, porque siempre en eso va haber trabajo, entonces cuando yo decidí entrar acá me dijo, yo no sé para qué quieres que te esté 
pagando tu carrera si de todos modos no vas a encontrar trabajo, te vas a morir de hambre. (S6,

E CICOM 1)

De acuerdo a la tercera idea, esta se centra en asociar, la licenciatura en CICOM con los medios de comunicación. En este sentido, los referentes inmediatos con los que se vincula a los estudiantes son imágenes populares propias de estos. Se trata de un desconocimiento sobre lo que en sí representa la carrera y sus diferentes ámbitos profesionales. Sin embargo, este imaginario se ve fortalecido con lo que Rizo (2012) señala como lastre de la comunicación en el campo educativo, el cual hace referencia a la indefinición del objeto de estudio en el propio campo dentro de los planes de estudio. Esto ha generado un socio-centrismo y media-centrismo que ha coadyuvado en gran medida a las múltiples concepciones de la profesión y a catalogarla, sobre todo, como sinónimo de medios.

\subsection{Ser estudiante de una universidad privada: implicaciones e imaginarios sociales}

En algún momento se consideró privilegiado a cualquier joven mexicano que cursara la educación terciaria, sin embargo, después de que incursionó el sector privado en este tipo de educación, los imaginarios sociales cambiaron. Por imaginario social se entiende de acuerdo a Pintos (2014) una imagen representativa que funge como un reflejo del pensar y de la práctica social. En este sentido, Suárez (2013) señala que a finales del siglo XX, el gobierno mexicano se ha encargado de fomentar la idea de que las escuelas de educación privada son mejores que las públicas; sin embargo, existen estudios que no sustentan esta idea, ni viceversa.

En este marco, los estudiantes señalan entre los imaginarios sociales más recurridos en su propio contexto cuatro ejes; el primero, enfocado a la calidad de las instituciones; el segundo, radica en el status económico de los alumnos; mientras que el tercero, se centra en la dependencia parental como un factor negativo para el desarrollo académico; y por último, el intelecto de los universitarios, mismo que a su vez se subdivide en una vertiente positiva y otra un tanto negativa, la primera considerándolos con un nivel intelectual alto, mientras que la segunda es centrada en considerar a los universitarios de escuelas privadas con nivel intelectual bajo.

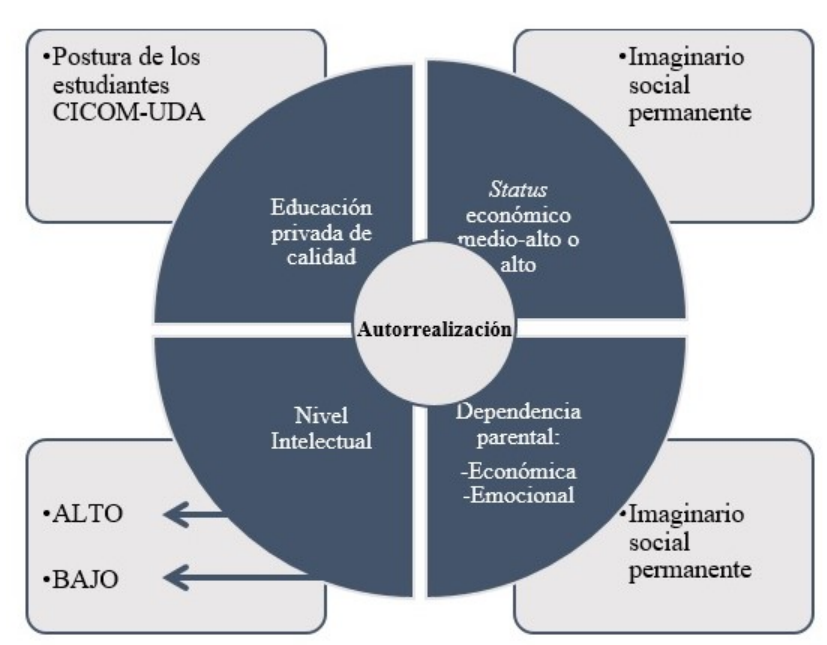

Figura 1. Imaginarios sociales sobre estudiar en universidades privadas. Fuente: elaboración propia

No obstante, es posible afirmar que también existe una postura neutral por parte de los informantes, la cual, se basa en el desempeño propio, es decir, la autorrealización, a continuación, se presenta un esquema que permite observar dichos imaginarios.

\subsection{Expectativas de éxito profesional}

En esta última categoría son presentados los hallazgos que componen tres ejes; el éxito profesional, los ámbitos profesionales y el campo laboral, los cuales, coadyuvaron a la correcta comprensión, interpretación y construcción de los resultados. En el primero, son presentados los factores que los estudiantes consideran importantes para alcanzar el éxito al egresar; el segundo, refiere a las diferentes líneas laborales en las que los alumnos buscan desempeñarse al concluir la universidad; y, por último, la percepción que tienen del campo laboral en cuanto a su profesión.

El éxito, más que un concepto, es para los estudiantes el referente de su futuro profesional, lo cual resulta lógico, no sólo por un posicionamiento de corte personal con relación a su porvenir, sino también por las altas expectativas que les proyecta la filosofía institucional, por ejemplo, la misión de la universidad reza su carácter de formadora de profesionales con calidad en sus servicios, es decir, se trata de una afirmación puntual de que la trayectoria de los estudiantes está resguardada por programas y servicios académicos que 
garantizan una estancia exitosa, además, de altas posibilidades de éxito profesional, aunque este, afirmaron, no sólo depende de la escuela, sino también de una mezcla de factores.

... lo que no importa tanto es la escuela donde vienes sino, cómo tú estás desarrollando tu trabajo después de que hayas salido de la universidad. (S2, E CICOM 5)

... depende de cómo lo explotes así tenga la mejor universidad privada (S5, E CICOM 5)

Para este punto es posible sostener que los alumnos consideran dos caminos principales para conseguir el éxito: el esfuerzo y la ruta autodidacta. En cuanto al primero, los actores son sabedores de que al egresar de la universidad deberán enfrentar una nueva etapa, pero que con esfuerzo lograrán la estabilidad laboral que esperan. Con relación al segundo, consideran necesario establecer una ruta autodidacta que deberán continuar, incluso, antes de concluir su licenciatura, para con ello, seguirse formando. Cabe mencionar, que son estudiantes que buscan desbordar su aprendizaje de lo que la universidad les ofrece durante su estadía en la misma.

En términos generales, la comunicación es un proceso que se encuentra en todo momento. En el campo laboral es posible numerar a los medios masivos de comunicación en los que se pueden desempeñar los especialistas en la materia. Sin embargo, no quiere decir que sean exclusivos de estos, es decir, existen diferentes ámbitos profesionales en los que pueden incursionar los futuros comunicólogos. En este sentido, los alumnos afirman que la carrera en Ciencias de la Comunicación posee un campo laboral muy amplio, en el cual es posible desenvolverse en diferentes ámbitos. No obstante, la mayoría de ellos refiere como un punto importante de éxito a los medios. Por ejemplo, el siguiente fragmento permite observar este posicionamiento consistente entre la voz de los actores:

...tengo pensado en [...] meterme al ámbito de YouTube o sea redes sociales y estar en televisión, hacer un programa, ser conductora de televisión, tenía pensado después de salir, bueno no exactamente después de salir, ¡eeh! entrar a
TV Azteca Puebla, o TV Azteca Monterrey o Guadalajara y empezar a meterme en ese ámbito de conducción. (S9, E CICOM 3)

En cuanto a lo señalado por los estudiantes es posible plantear un análisis no sólo desde la voz de estos, sino también desde la realidad institucional. Se trata de alumnos que colocan una visión laboral en medios de comunicación, esto en consistencia con los planes curriculares que la misma institución oferta. Además de que la UDA surge como una universidad pionera en la materia, aunque en sus inicios coloca un énfasis particular en periodismo, a través de los años ha buscado que sus estudiantes se coloquen en diversos medios. De este modo, la mayoría de los alumnos buscan seguir esa ruta heredada desde una tradición institucional. No obstante, hay quienes escapan de esta mirada y colocan sus aspiraciones en otros horizontes, propios de la comunicación pero no exclusivos de medios.

Cuando se trata de campo laboral, los estudiantes plantean tres condicionantes: prestigio institucional, económico-sociales y geográficos; siendo las tres importantes para su incursión en el campo laboral al concluir su licenciatura. De manera general, la primera refiere a las posibilidades de conseguir un empleo en cuanto al posicionamiento de la institución que egresan, mientras la segunda, aunada a la primera, se enfoca en la posibilidad de obtener un primer empleo con el imaginario permanente sobre el nivel socioeconómico que se les atribuye al ser parte de una universidad privada. En cuanto a la tercer condicionante, en esta señalan las escasas posibilidades de conseguir un empleo en medios de comunicación dentro de la entidad.

\section{Resultados}

Esta investigación, tuvo como intención el poder conocer quiénes son los estudiantes de la licenciatura en Ciencias de la Comunicación de la Universidad del Altiplano, además de comprender cómo conforman su identidad universitaria e identificar los imaginarios existentes en cuanto a su condición de estudiantes de una institución privada, y en particular, como estudiantes de este programa educativo. Finalmente, se buscó analizar las expectativas de éxito profesional que construyen durante su trayectoria escolar, todo ello, en consistencia con el posicionamiento epistémico asumido, a partir del 
estudio de los universitarios en su temporalidad, espacialidad, corporalidad y relacionalidad.

Los hallazgos, no sólo permitieron alcanzar estas intenciones de generación de conocimiento, ya que estas fueron superadas, mediante el contacto con los entrevistados quienes compartieron sus palabras y con estas, su sentir como parte de esta comunidad.

En cuanto a la preocupación de los alumnos de primer cuatrimestre, estos colocaron un énfasis en dos ejes; el principal soportado en la identidad universitaria y otro, un tanto más secundario, pero en la misma línea, en la identidad CICOM, lo cual, resulta un tanto consistente, pues son alumnos de nuevo ingreso quienes buscan, en gran medida, sentirse parte de lo que es ahora su nueva institución.

Por otra parte, para los alumnos de tercer cuatrimestre su preocupación fue colocada más en lo que corresponde a la vida universitaria, en este sentido, se trata de alumnos que ya cuentan con poco más de un año en la institución, quienes buscan una vida cultural o deportiva que contribuya a la construcción de una identidad universitaria.

De igual forma, los estudiantes de quinto cuatrimestre enfatizan una preocupación en la vida universitaria, pues después de dos años, buscan fomentar una vida cultural en la universidad, además, que otro eje al que también refieren con frecuencia es, la identidad CICOM, pues a la mitad de su carrera, sostienen que la elección de vida es acertada y que plenamente se identifican con su programa educativo.

A diferencia de los otros cuatrimestres, los alumnos de séptimo colocan mayor importancia en dos ejes; sobre todo, en las expectativas de éxito profesional, lo cual resulta consistente pues están en la parte final de su licenciatura y a meses de incursionar en el campo laboral, sin embargo, los imaginarios sociales sobre la condición de estudiante de escuela privada y sobre el comunicólogo, también permanecen como una preocupación constante en ellos.

Si bien, los alumnos sujetos de estudio fueron de diferentes cuatrimestres, es posible ver las principales preocupaciones de cada grupo, lo que resulta consistente con el grado que cursan, su estadía universitaria, incluso, con el plan curricular bajo el cual ingresaron a la universidad.

Con todo ello, es posible señalar que los alumnos construyen una identidad soportada en su adscripción al programa educativo y no tanto a la institución. Además de que algunos factores de identidad de la propia institución no coadyuvan a la creación de este vínculo universidad-alumno, pues tan sólo, en cuanto al lema, refieren a este como burla, incluso, lo asocian a una canción popular que lejos de promover el arraigo, resulta ser un factor negativo en la comparación con otras universidades. En este sentido, y en consistencia con los fundamentos epistémicos, la presente investigación recuperó la esencia de cómo los estudiantes viven su mundo universitario y de cómo lo piensan, además de buscar la comprensión de sus experiencias a partir de su propio contexto, en la Universidad del Altiplano.

\section{Referencias}

Basaldúa, E. et al., (2013). El sentido del proceso educativo de los estudiantes de la IBERO Puebla. México: Aru.

Castoriadis, C. (2002). La insignificancia y la imaginación. Diálogos con Daniel Mermet, Octavio Paz, Alain Finkielkraut, Jean-Luc Donnet, Francisco Varela y Alain Connes. Madrid: Trotta.

Centro de Investigación para la Paz, FUHEM (2005) Culturales e identidades. Recuperado de http://www. fuhem.es/ecosocial/dossierintercultural/contenido/9\% 20EL\%20CONCEPTO\%20DE\%20IDENTIDAD.pdf

De Garay, A. (2012) Mis estudios y propuestas sobre los jóvenes universitarios mexicanos. México: Unión de Universidades de América Latina y el Caribe.

Díaz-Barriga F. y Hernández G. (2002). Estrategias docente para un aprendizaje significativo. Una interpretación constructivista. México: McGraw-Hill.

Giddens, A. (2002) Modernidad e identidad del yo: el yo y la sociedad en la época moderna. Brasil: Editora Zahar.

Giménez, G. (1997) Materiales para una teoría de las Identidades Sociales. Recuperado de http://www.gime bez.com.mx/index.html

Larrain, J. (2003). El concepto de identidad. Revista FAMECOS (21) Recuperado de http://revistas.univercie ncia.org/index.php/famecos/article/viewfile/348/279

Lozano, A. y Rodríguez, M. (2005). Perfil de ingreso: Serie histórica 1995-2003 (Estudios sobre la UPN). México: Colección Más Textos, no. 2. Universidad Pedagógica Nacional.

Pintos, J. (2014). Algunas precisiones sobre el concepto de imaginarios sociales. Revista Latina de Sociología. (4) Recuperado de http://ruc.udc.es/dspace/bitstream/hand le/2183/14482/RLS_2014_4_art_1.pdf?sequence=1

PROCESO (2016). México es tercer lugar mundial de periodistas asesinados en 2016: FIP. Recuperado de 
http://www.proceso.com.mx/468158/mexico-tercer-lu gar-mundial-periodistas-asesinados-en-2016-fip

Rama, S. (2011). Professor's performance for effective teaching (Kosovo case). Procedia Social and Behavioral Sciences. Volumen 12. (Pp. 117-121).

Rizo, M. (2012) Imaginarios sobre la comunicación. Algunas certezas y muchas incertidumbres en torno a los estudios de comunicación, hoy. España: Instituto de la Comunicación, Universidad Autónoma de Barcelona.

Sartorello, S. (2013). Identidades estudiantiles y relaciones interculturales en la UNICH. En S. Sartorello y T. Cruz (coordinadores). Voces y visiones juveniles: en torno a diversidad, diálogo y conflicto intercultural en la UNICH. México: FONDEIB.

Suárez, M. (2013). Educación superior pública y privada en México. Desigualdades institucionales y opiniones de los estudiantes. Serie: Cuadernos Digitales del Seminario de Educación Superior de la UNAM, No. 4. México: SES, UNAM. Recuperado de http://www.ses. unam.mx/integrantes/uploadfile/hsuarez/Suarez_Educa cionSupPublicaYPrivada.pdf 